\title{
Spectral envelope control for a flat frequency comb spectrum
}

\author{
Michael Debus $\odot,{ }^{\text {a,* }}$ Philipp Huke $\odot,{ }^{\text {a,b }}$ David Meyer, ${ }^{\text {a }}$ and \\ Ansgar Reiners $\oplus^{a}$ \\ ${ }^{\mathrm{a}}$ Georg-August-Universität, Institut für Astrophysik, Göttingen, Germany \\ bInstitute for Lasers and Optics, Hochschule Emden/Leer, Emden, Germany
}

\begin{abstract}
Laser frequency combs have properties that make them promising spectrograph calibration light sources. One drawback for this application is the high dynamic range in the supercontinuum spectra of some frequency combs. We aim to flatten the spectrum of a Ti:sapphire laser frequency comb to improve the calibration performance for a Fourier transform spectrograph. For this, we develop a compact Fourier transform optical pulse shaping setup, which enables control of the spectral envelope via dispersion of the light onto a spatial light modulator (SLM). We demonstrate that this setup allows us to flatten the comb spectrum in the wavelength range 780 to $980 \mathrm{~nm}$. For $90 \%$ of the wavelength range, the dynamic range is below $3 \mathrm{~dB}$. In the flattened spectra 3\% more comb lines can be detected. The line center fit precision, however, is reduced due to noise caused by the SLM. (c) The Authors. Published by SPIE under a Creative Commons Attribution 4.0 Unported License. Distribution or reproduction of this work in whole or in part requires full attribution of the original publication, including its DOI. [DOI: 10.1117/1.JATIS.7.2.025005]
\end{abstract}

Keywords: laser frequency comb; spatial light modulator; spectral flattening; supercontinuum.

Paper 21016AS received Feb. 1, 2021; accepted for publication May 20, 2021; published online Jun. 3, 2021.

\section{Introduction}

Laser frequency combs (LFCs) provide evenly spaced narrow emission lines that cover a broad spectral range. Their spacing and position can be controlled to a very high accuracy and precision. These properties make LFCs suitable wavelength calibration light sources for astronomical spectrographs. ${ }^{1,2}$

However, there are a number of challenges involved in creating such an astro-comb. ${ }^{3}$ To cover a large spectral range, a broadening of the LFC spectrum in a nonlinear fiber is often required. ${ }^{4,5}$ The resulting broadened spectrum exhibits strong intensity variations. Furthermore, the spectral envelope changes over time as the nonlinear broadening process is very sensitive to changes in the peak pulse power and polarization. ${ }^{3}$ This can lead to over- or under-exposures in calibration measurements of a spectrograph as well as wavelength errors in the calibration process.

As a solution to this problem, the use of a Fourier transform optical pulse shaping setup has been established. ${ }^{6,7}$ In such a setup, the light is spectrally dispersed onto a spatial light modulator (SLM) ${ }^{8,9}$ The pixels of an SLM can individually manipulate incident light in amplitude, phase, or polarization. ${ }^{10}$ This enables control of the power in different wavelength regions.

Our aim is to flatten an LFC spectrum to improve its calibration performance for a Fourier transform spectrograph (FTS), but the same approach is also suitable to calibrate an astronomical echelle spectrograph. We base our setup on Ref. 7 with adjustments for different characteristics of our LFC and requirements of our setting. We develop a compact optical setup, which allows for strong attenuation while minimizing the background.

*Address all correspondence to Michael Debus, mdebus@phys.uni-goettingen.de 


\section{Setup}

\subsection{Design Considerations}

Our aim is to use an LFC with a flattened spectrum as a calibration light source for an FTS. We want to be able to perform simultaneous measurements of the LFC and solar light with the FTS. From this we formulate the requirements for our spectral flattening setup.

The spectrum of the LFC we use has a strong variability in power of more than $20 \mathrm{~dB}$ (see Fig. 1), resulting in low signal-to-noise-ratio (SNR) for some wavelength ranges in our FTS measurements. Fit uncertainty increases dramatically for comb lines with an SNR lower than 3 and is much improved at an SNR of $20 .{ }^{11}$ From these results, we can see that we want the dynamic range in the comb spectrum to be $15 \mathrm{~dB}$ at most, although $<7 \mathrm{~dB}$ would be ideal. To not cover the solar wavelength range of interest below $700 \mathrm{~nm}$, we choose a range of 700 to $1000 \mathrm{~nm}$ for our spectrum control setup. Furthermore, the spectrum should stay constant for at least 16 min, i.e., the time of a single measurement at maximum resolution. Ideally, multiple measurements with the same spectrum should be possible.

An auxiliary requirement is that we want to minimize the size of the setup. The LFC delivers roughly $25 \mathrm{~mW}$ of linearly polarized light in the spectral range of 690 to $1000 \mathrm{~nm}$ to the setup. As this is enough power for our purposes, we can use the optical grating in a non-Littrow configuration. $^{12}$ We opted for a forward-backward design, where the light is coupled in and out through the same polarizing beam splitter (PBS). This improves optical quality at the cost of efficiency. This design is fairly easy to align while also reducing the space required for the setup.

\subsection{Setup Description}

A schematic of the setup is shown in Fig. 2. The linearly polarized light from the LFC is coupled in at a PBS where it is reflected. It is then dispersed at an optical grating and the first order of refraction is focused onto the SLM by a cylindrical lens. Each pixel column of the SLM can now individually rotate the polarization state of a different wavelength interval. The light is reflected back and collimated by the cylindrical lens. It is then recombined by the grating. Both the SLM and the grating are in the focal plane of the cylindrical lens. From the grating, the light is reflected back on the PBS, where the fraction of the light with changed polarization now passes in transmission. The light that passes through the PBS is then further split up by a non-PBS. Since this beam splitter has slightly polarization-dependent reflection and transmission coefficients, we rotate the nominal 90:10 beam splitter to achieve a 95:5 split ratio. The 5\% part of the light is coupled into a hexagonal fiber leading to a spectrometer. The light measured at the spectrometer is used to control the feedback loop with the SLM, ensuring the shape of the spectrum is as desired. The $95 \%$ part of the light is the final output.

Our full setup, including an enclosing box, as well as the spectrometer and SLM driver unit fits on a $300 \times 500 \mathrm{~mm}$ breadboard. Based on Zemax simulations, we presume that using smaller optics and mounts the optical elements could fit on an area of $100 \times 200 \mathrm{~mm}$.

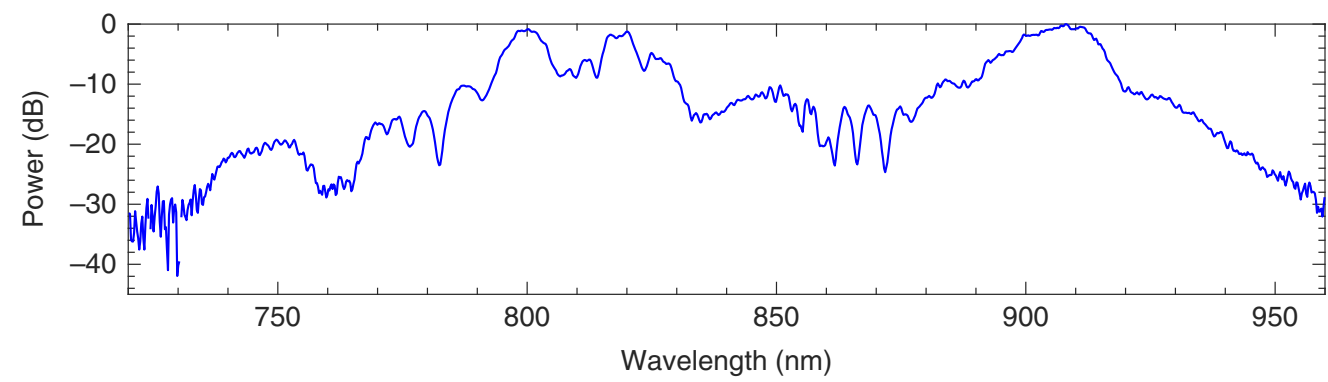

Fig. 1 A typical LFC spectrum, exhibiting a high dynamic range. Intensity changes of more than $10 \mathrm{~dB}$ within $<5 \mathrm{~nm}$ as well as a lot of small-scale structure throughout the whole spectrum can be seen. 


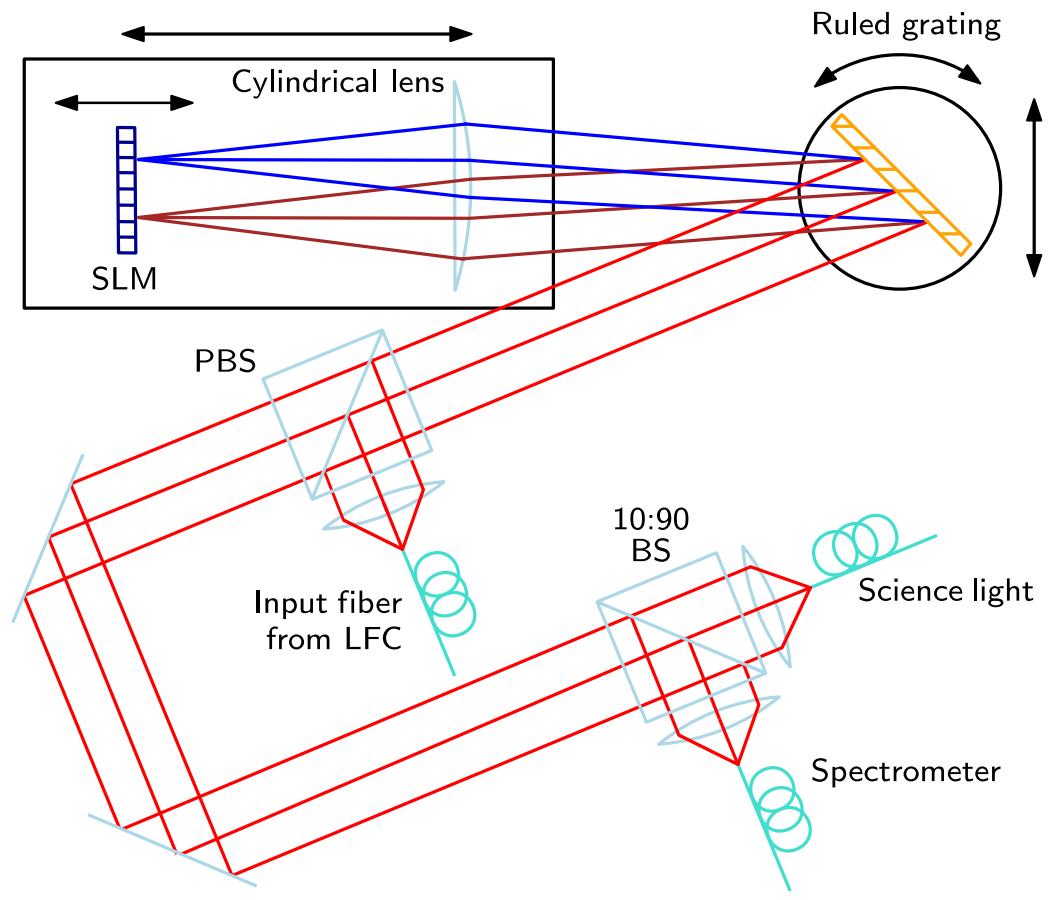

Fig. 2 Schematic drawing of the spectrum control setup. Red lines indicate combined light, dark red, and blue lines indicate dispersed light. Straight arrows indicate linear stages, the curved arrow indicates a rotation mount.

\subsection{Hardware}

\subsubsection{Laser frequency comb}

The type of LFC is a Ti:Sapphire laser with a repetition rate of $1 \mathrm{GHz}$, i.e., the spacing of the comb lines is $1 \mathrm{GHz}$. The light from the laser is spectrally broadened in a nonlinear polarizationmaintaining fiber (NL-PM-750) with a length of $0.5 \mathrm{~m}$. An example of the spectral envelope of the comb is shown in Fig. 1. It has a very high dynamic range. The spectrum is not stable over time, as slight changes in the coupling to the nonlinear fiber, e.g., through vibrations or temperature changes, lead to changes of the spectral envelope. The overall usable output power after the nonlinear fiber strongly depends on the coupling as well. Typically, about $25 \mathrm{~mW}$ of linearly polarized light in the desired wavelength range are coupled into the spectral flattening setup with a polarization-maintaining single-mode optical fiber (PM780-HP).

\subsubsection{Spatial light modulator}

The SLM in our setup is the model HED 6001-NIR (Holoeye Photonics), which is a liquid crystal on silicon display. Each pixel of the display is a cell filled with liquid crystals of an ellipsoidal shape. If a voltage is applied to a cell, the crystals change their orientation and thereby the birefringence of the pixel, causing a phase shift, similar to a wave plate. The magnitude of the phase shift can be controlled as it depends on the applied voltage.

The SLM has $1920 \times 1080$ pixels with a pixel pitch of $8 \mu \mathrm{m}$ and a fill factor of $93 \%$. The voltages applied to the pixels can be set to 256 different levels, called gray values. For simplification, we will refer to the gray values as pixel values. In the driver unit of the SLM, a look-uptable is stored, which specifies the voltage corresponding to each pixel value. This look-up-table is called the gamma curve. At $830 \mathrm{~nm}$, the achievable contrast ratio is specified to be better than $400: 1$ while the reflectivity is $64 \%$. The frame rate of the display is $60 \mathrm{~Hz}$, with a typical response time of 9 to $18 \mathrm{~ms}$. 


\subsubsection{Further optical elements}

The focusing lens is a cylindrical achromat with a focal length of $75 \mathrm{~mm}$. The reflective ruled grating has 600 lines $/ \mathrm{mm}$ and a blaze angle of $17.6 \mathrm{deg}$. The light has an incidence angle of about $24.5 \mathrm{deg}$ on the grating, which leads to a resolving power of about 2400 , a linear dispersion on the SLM of about $0.177 \mathrm{~nm} /$ pixel and an available bandwidth of about $340 \mathrm{~nm}$. The central wavelength can be adjusted by turning the grating on the rotation mount.

\subsubsection{Spectrometer}

We use the BlueWave-NIR2-25 (StellarNet) spectrometer, which covers the wavelength range from 590 to $1020 \mathrm{~nm}$ with a minimum exposure time of $1 \mathrm{~ms}$. It has a nominal resolution of $0.5 \mathrm{~nm}$ FWHM. Ideally, the resolution should be slightly better since the linear dispersion on the SLM is $0.177 \mathrm{~nm} /$ pixel. However, the image of the incoupling fiber on the SLM-assuming monochromatic light— has a size of roughly $25 \mu \mathrm{m}$, corresponding to about 3 pixels of the SLM. Thus, a bandwidth of about $0.55 \mathrm{~nm}$ is incident on a single pixel, which matches the spectrometer resolution.

\subsubsection{Fourier transform spectrograph}

The FTS we aim to calibrate is a commercial instrument (Bruker Optics, IFS 125HR) with a maximum resolving power of about $2.8 \cdot 10^{6}$ covering the wavelength range from 500 to $1300 \mathrm{~nm}$. The FTS setup is described in more detail in Ref. 13. It is located in a different laboratory than the LFC, so the light from the spectral flattening setup is transported using a $100 \mathrm{~m}$ long multimode fiber (NA 0.22, core diameter $200 \mu \mathrm{m}$ ), which is then butt-coupled to the hexagonal input fiber (CeramOptec, NA 0.26 , core width $525 \mu \mathrm{m}$ ) of the FTS.

\subsection{Efficiency}

The achievable efficiency according to the specifications of the components of our setup is shown in Table 1. At a wavelength of $830 \mathrm{~nm}$, the possible efficiency is $24.9 \%$, whereas at

Table 1 Efficiencies of the different optical components in percent at 830 and $1064 \mathrm{~nm}$. Grating efficiencies are given for Littrow configuration only.

\begin{tabular}{lcc}
\hline \hline Component & $830 \mathrm{~nm}$ & $1064 \mathrm{~nm}$ \\
\hline Collimator & $>97.5$ & $>97.5$ \\
PBS p-pol & 99.5 & 99.5 \\
Grating p-pol & 72.0 & 88.0 \\
Lens, coated & 99.5 & 99.5 \\
SLM & 64.0 & 78.0 \\
Lens, coated & 99.5 & 99.5 \\
Grating s-pol & 64.0 & 51.0 \\
PBS s-pol & 97.0 & 98.0 \\
Mirror & 98.9 & 98.5 \\
Mirror & 98.9 & 98.5 \\
$90: 10$ BS s-pol & 95.0 & 95.0 \\
Collimator & $>97.5$ & $>97.5$ \\
Overall & $>24.9$ & $>29.6$ \\
\hline \hline
\end{tabular}


$1064 \mathrm{~nm}$ it is $29.6 \%$. As efficiencies of the grating were only supplied for the Littrow configuration the actual efficiencies attainable in our configuration are lower.

\subsection{Calibration of the Spectral Flattening Setup}

The calibration process consists of three steps. First, we measure the transmitted power as a function of wavelength and voltage applied to the pixels of the SLM. Second, we calibrate the wavelength, i.e., determine which wavelength is incident on which pixel column. Finally, we want the output spectrum to be at the set point for the FTS and not for the spectrometer. Thus, we have to cross calibrate both instruments, to account for the difference in spectral efficiency between the spectrometer and the FTS as well as possible wavelength-dependent absorption of intermediate optical components.

\subsubsection{Power calibration}

For the power calibration process, we take three spectrometer exposures in direct succession for each pixel value. The first is an exposure with the current pixel value $p$. The second is a dark exposure with all pixels set to zero to subtract the background. The last one is a flat exposure, with all pixel values set to a level that roughly corresponds to the maximum intensity for the central wavelength. For our setup, we use a pixel value of 230 for a central wavelength of $900 \mathrm{~nm}$. We use the LFC as the light source for this measurement. Due to its spectral intensity fluctuations, the flat exposure becomes necessary. For a stable light source, a single exposure for each pixel value would suffice. From the calibration measurements, we calculate the spectral intensity response using

$$
I(\lambda, p)=\frac{I_{p}(\lambda)-I_{\mathrm{dark}}(\lambda)}{I_{\mathrm{flat}}(\lambda)-I_{\mathrm{dark}}(\lambda)}
$$

The pixel value for which the intensity is maximal, changes with wavelength. Thus, we normalize the spectral intensity response by dividing by the respective maximum value of $I$ for each wavelength

$$
I_{\text {norm }}(\lambda, p)=\frac{I(\lambda, p)}{I_{\max }(\lambda)} .
$$

In the next step, we translate the pixel values to voltages using the gamma curve. The gamma curve specifies the voltage applied to each pixel for each pixel value input. It is stored as a lookup-table in the driver unit of the SLM.

For our setup, we want the spectral intensity curve to be linear with respect to pixel value, as we use a proportional-integral-derivative-controller (PID-controller) to manipulate the spectrum. Furthermore, we want to optimally use the available range of pixel values. For this purpose, we redefine the gamma curve. We set the voltage corresponding to the highest pixel value to $V_{\text {high }} . V_{\text {high }}$ is the maximum of the voltages for which maximum intensity is reached for each wavelength in the given range $\left(V_{\text {high }}=\max \left\{V\left(I(\lambda)=I_{\max }(\lambda)\right)\right\}\right)$. The pixel value of zero corresponds to zero voltage as this gives the lowest intensity. The remaining pixel values are set to voltages such that the intensity response becomes linear with respect to pixel value. ${ }^{14}$ The response is then also sufficiently close to linear for the neighboring wavelengths. The deviation from a linear response with an optimized gamma curve is typically lower than 5\% and does not exceed $12 \%$ (see Fig. 3). For lower wavelengths, a drop in intensity at high pixel values is observed.

\subsubsection{Wavelength calibration}

The aim of the wavelength calibration is to determine which wavelength range is incident on which pixel column. We refer to pixel columns on the SLM as the lines of pixels perpendicular to the dispersion direction of the light. We switch on individual pixel columns, i.e., set them to a 


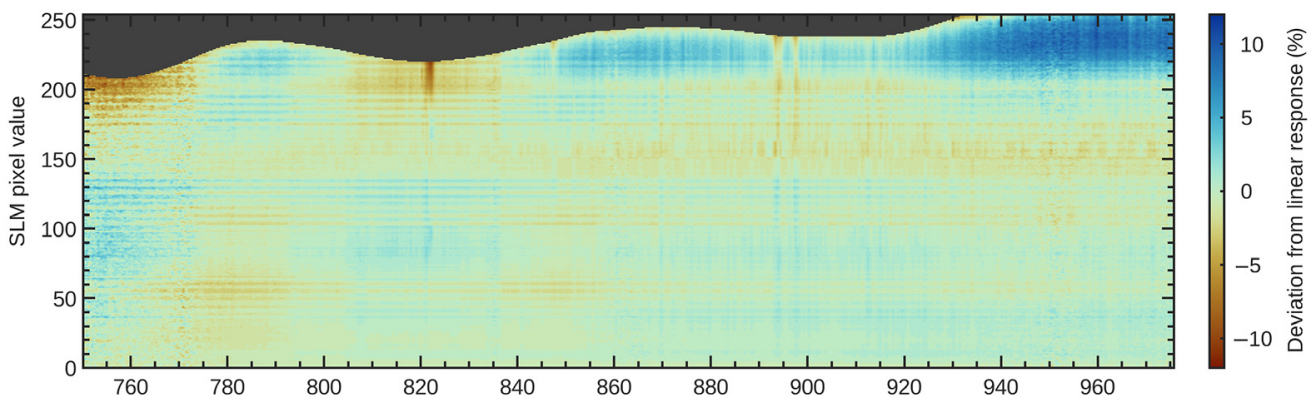

(a)

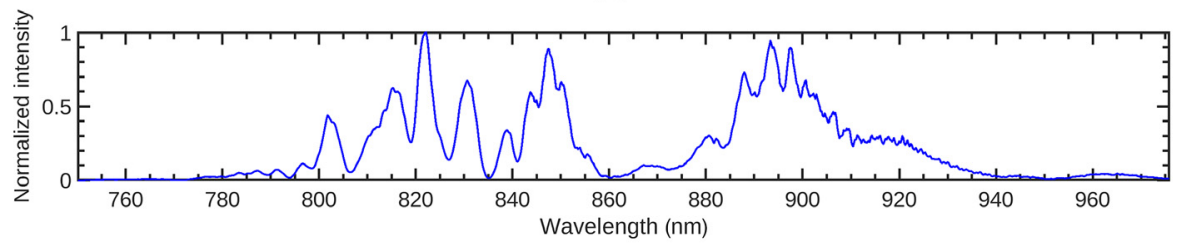

(b)

Fig. 3 (a) Deviation from a linear curve for each wavelength and pixel value. In the black region in the top left, the intensity drops below the maximum. The horizontal lines are caused by digitization effects in the addressing of the SLM pixels. The vertical lines in (a) correspond to peaks in the intensity spectrum and are caused by intensity variations in the calibration spectrum (see bottom panel). (b) Normalized intensity of the calibration light source spectrum.

high pixel value, whereas the other pixels are set to a pixel value of zero. The measured spectrum then displays a peak in the wavelength range, which is incident on the enabled pixel column.

As before, we take three exposures of equal length for each measurement. The first exposure is with the individual pixel column set to the pixel value corresponding to the maximum intensity of the central wavelength. The second is a dark exposure with all pixels set to zero. Lastly, we take a flat with all pixels set to a such a pixel value that the count rate is maximized without risk of overexposure. Each measured spectrum is flat- and dark-corrected and the peaks are fitted to obtain the wavelength position. Finally, a third-degree polynomial is fitted to the measured pixelwavelength curve, yielding the wavelength solution (see Fig. 4). The systematics that can be seen in the residuals are caused by the spectral variability of the comb in these wavelength ranges.

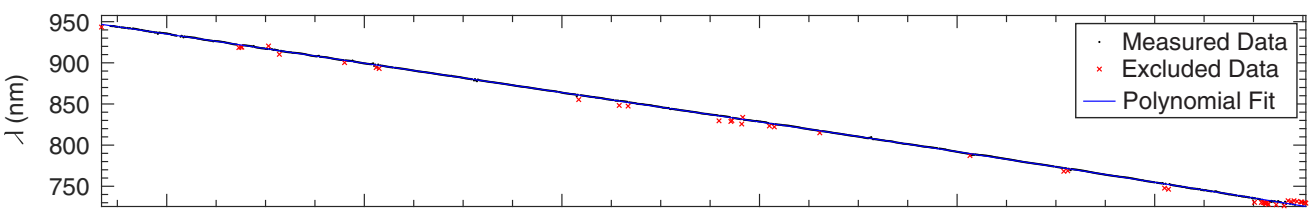

(a)

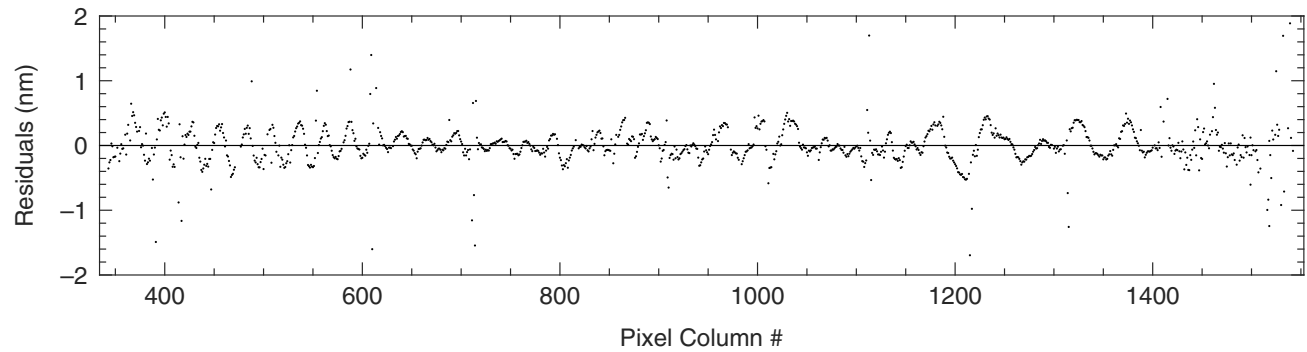

(b)

Fig. 4 The measured peak positions of activated pixels (black dots) and the third-degree polynomial fit (blue line) are shown in (a). Outliers not included in the fit are indicated by red crosses. (b) The fit residuals. 
To speed up the calibration process, instead of switching on a single-pixel column per measurement, we switch on multiple pixel columns in regularly spaced intervals of 100 pixels. The interval is chosen large enough so that the peaks in the recorded spectrum are well separated. In each subsequent measurement, the activated pixel columns are shifted one pixel to the right. This is repeated 99 times so that each pixel column is activated once.

The spectrum of the LFC does not cover the full display of the SLM. Therefore, we cannot identify the edges of the SLM in the measured spectrum. Thus, we are not able to directly match a peak in the spectrum to a specific activated pixel column. To do this, we create a preliminary wavelength solution. We perform two measurements, each with a single-pixel column switched on. We choose columns from the two opposing ends of the illuminated surface. The linear curve connecting the two data points is the preliminary wavelength solution that enables matching each peak to the corresponding active pixel column. The equally spaced peaks from the previous measurements already define the slope of the full wavelength solution. In principle, a single exposure with only one activated pixel column would therefore suffice to anchor the wavelength solution. However, we found a preliminary wavelength solution using two exposures to give more stable results so that we make this one additional measurement.

\subsubsection{Cross calibration}

Due to the different measurement principles of the FTS and the spectrometer, as well as possible wavelength-dependent absorption in the transport from the spectrum control setup to the FTS, the same spectrum measured by the two instruments will differ. To achieve the desired response in the FTS rather than the spectrometer, which controls the spectrum, we perform a cross calibration of the intensity. ${ }^{7}$ We activate the control loop and set it to a flat spectrum and perform simultaneous measurements in the FTS. We then fit the spectral envelope as measured by the FTS. The inverse of the fit can now be multiplied with the spectral set point of the control loop so that we get the desired response in the FTS.

\subsection{Control Loop}

We set up a control loop that adjusts the pixel values of the SLM pixel columns so that the output spectrum has the desired form. We implement the control loop in LabVIEW using the built-in multivariable PID-controller function. Using the wavelength solution, we translate the wavelengths in the measured spectra to pixels. The spectra are then interpolated at integer values. The same is done with the desired reference spectrum. In each iteration, the PID-controller uses the measured and reference spectra to compute the adjusted pixel values for each pixel column. The maximum pixel value for each pixel column is fixed to the value of maximum intensity for the corresponding wavelength (i.e., the black region in Fig. 3 is not accessible). This ensures that the control loop cannot overshoot and thus decrease output power when trying to increase it. Furthermore, we smooth the control output with a kernel corresponding to the instrumental line shape (ILS) on the SLM.

\section{Results}

\subsection{Spectrum Control}

With the spectral flattening enabled, we recorded ten interferograms of the LFC with the FTS while simultaneously measuring the output with the spectrometer (see Fig. 5). We are able to flatten the wavelength range 780 to $980 \mathrm{~nm}$ to within $6 \mathrm{~dB}$, with $96 \%$ of the range better than $3 \mathrm{~dB}$ in the spectrometer measurements. The FTS measurements also show a flat spectral envelope and reduced dynamic range, with $90 \%$ within $3 \mathrm{~dB}$ of the set point. Compared to the spectrometer measurements, more small-scale structure becomes apparent in the high-resolution spectra. We are able to detect about 3\% more comb lines in flattened spectra compared to spectra where the control loop was disabled. However, the line fit precision is decreased by $8 \%$ on average if the control loop is enabled. 


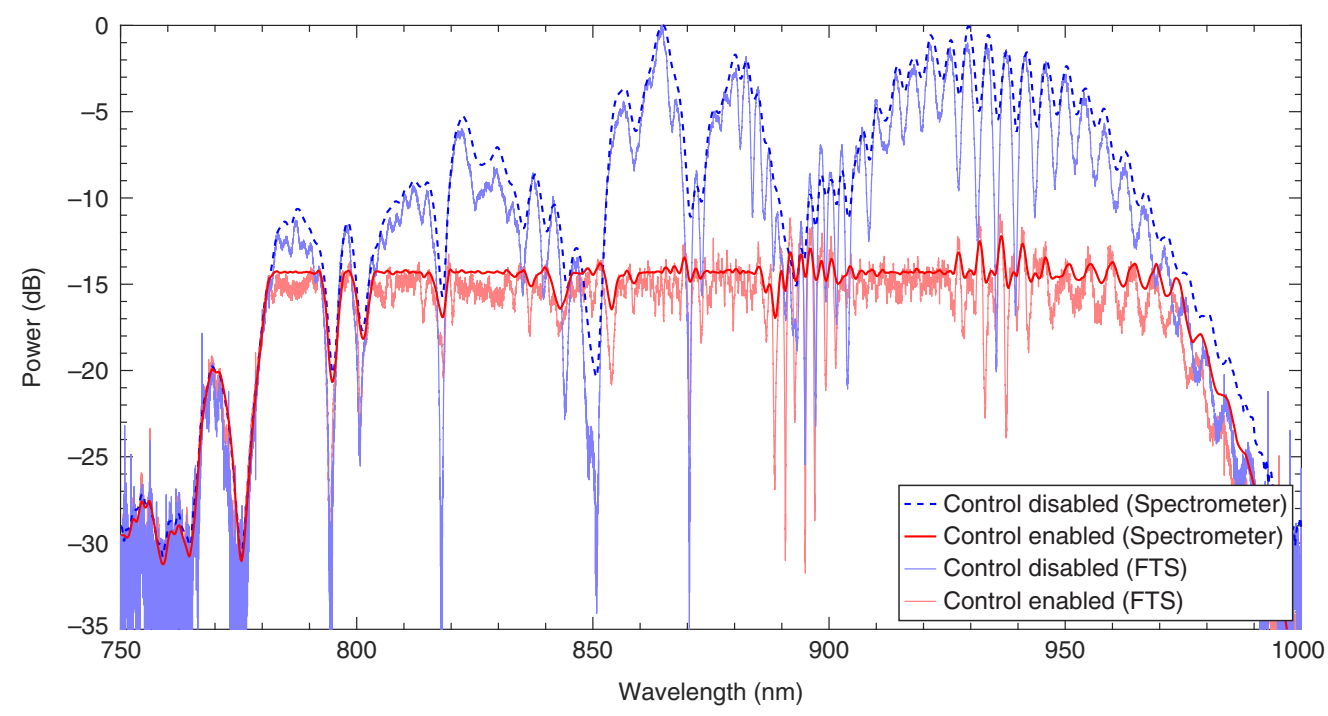

Fig. 5 Measured LFC spectrum with control disabled (blue lines) and control enabled (red lines). The thick lines correspond to the spectrometer measurements, and the weaker, thin lines are FTS measurements. In the FTS measurements only the spectral envelope is shown for better visibility.

The decreased fit precision is linked to the operating principle of the SLM. The voltage applied to an SLM pixel over a single frame does not stay constant but follows a distinct modulation pattern depending on the pixel value. We found that this modulation causes artificial peaks in the spectrum. They are visible in the spectral regions from 750 to $770 \mathrm{~nm}$ and 990 to $1000 \mathrm{~nm}$ (see Fig. 5) but masked at higher optical intensities. Furthermore, the modulation significantly raises the noise floor of the measurements causing the reduced line fit precision. The amplitude of the modulation is stronger at intermediate pixel values, whereas it becomes weaker for pixel values close to zero or the maximum. Therefore, the noise is reduced when the SLM is set to maximum throughput compared to when the control loop is active. Due to systematics introduced by this SLM modulation, a phase correction of the interferograms could not be performed.

The control loop remained stable over time. We did not see any signs of a break down over a span of more than $4 \mathrm{~h}$.

\subsection{Instrumental Line Shape}

We measured the ILS of the spectral flattening setup, i.e., the spectral response of a single activated pixel column (see Fig. 6) using the FTS and the spectrometer. We compare the measured ILS with an analytical model of the spectral response, ${ }^{15}$ assuming a gaussian light distribution exiting the single-mode incoupling fiber with the specified mode field diameter. The analytical model is in agreement with the FTS measurement, indicating a good alignment of the setup (see Fig. 6).

A comparison between the measurements of the spectrometer and the FTS shows that the spectrometer falls short of its nominal resolution by a factor of about three. The spectrometer ILS is skewed and exhibits a strong tail toward higher wavelengths from the line center. This explains why the spectrometer measurements match the FTS measurements more closely on the low wavelength side of peaks than on the high wavelength side (see Fig. 5).

\subsection{Efficiency}

The overall efficiency of the setup is $17.2 \%$ if the SLM is set to maximum throughput. This is less than the values calculated in Sec. 2.4, as the incoupling angles at the grating are $24 \mathrm{deg}$ and $5 \mathrm{deg}$ on the way in and out, respectively. The efficiency is only given for the Littrow configuration with an incoupling angle of $17.6 \mathrm{deg}$, and we thus expect the efficiency to be significantly lower. The throughput drops to about $2 \%$ to $4 \%$ if the spectral flattening is turned on. The value 


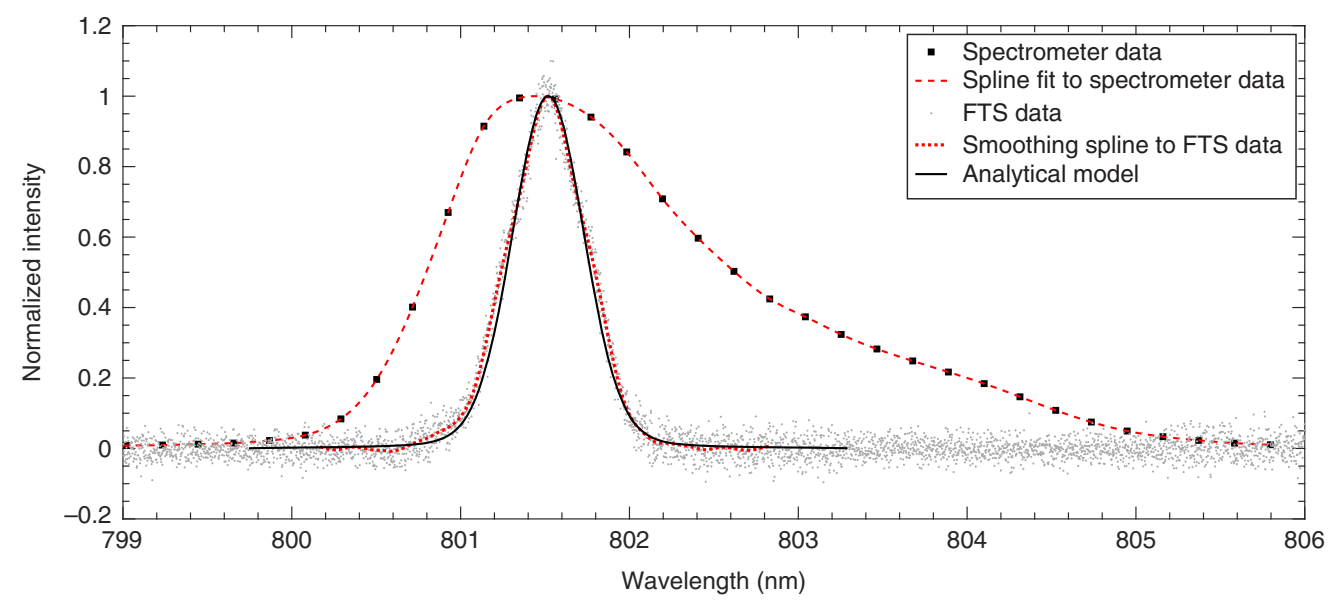

Fig. 6 Measured intensity response for a single activated pixel column, i.e., the ILS of the spectrum control setup. A spectrometer measurement (black squares) and a corresponding spline fit (red dashed line), as well as an FTS measurement (gray dots), a corresponding smoothing spline fit (red dotted line) and an analytical model of the intensity response (black line) are shown.

depends on the specific spectrum, as a lot of the loss factors, particularly the SLM and grating efficiency, are wavelength dependent.

\section{Discussion}

We show that the spectrum control setup works as desired, by reducing the dynamic range and temporal stability. The results from the spectrometer measurements are confirmed by highresolution FTS spectra. A higher number of comb lines can be fitted in the flattened spectra. However, the intensity modulation in each SLM frame is detrimental to the line fit quality in the FTS measurements. Unfortunately, the modulation becomes more pronounced when the spectrum control is enabled as intermediate pixel values cause stronger modulations. An astronomical echelle spectrograph would not suffer from these effects, as the measurements are typically averaged over timescales substantially longer than the duration of a single SLM frame (17 ms). Calibration of an echelle spectrograph could therefore be improved using the spectrum control setup and the resulting reduction in dynamic range and spectral variability of the comb spectrum.

For calibration measurements, we have to make a trade-off between flatness, bandwidth, and throughput. The radial velocity information contained in a single line only slightly depends on the intensity as long as the SNR is above 3 in the FTS measurements. ${ }^{11}$ We, therefore, place a higher value on bandwidth, which increases the number of detectable lines. A higher throughput reduces amplifier noise but in our case, this is not a driving factor in trade-off considerations.

The spectral envelope stability in our setup is limited by the low resolution of the spectrometer. This limits the wavelength calibration precision of the spectrum control setup. More importantly, the spectrometer cannot resolve the structure in regions with a high dynamic range on short-wavelength scales. This leads to systematic errors in the control loop, which are responsible for the residual modulation of the flat spectrum. To amend this, we smooth the control output, which improves the flatness but some deviations from a flat spectral envelope remain. As shown by the control setup ILS measurements, a spectrometer with a higher resolution is required to fully take advantage of the capabilities of the setup.

The limiting factor for the spectral bandwidth that can be flattened is the initial dynamic range in combination with the spectral contrast ratio. This limits how low we can set the intensity level in the control loop. The lower we set the overall intensity, the broader the flat top of the spectral envelope. We usually set the initial value of the plateau so that about $4 \%$ of the pixel value control range remains at the lowest points. This way, the control loop can still react to changes of the spectral envelope. 
The largest improvement for the throughput efficiency would be a flatter initial spectrum so that we can operate closer to the $17.2 \%$ of maximum throughput. The initial spectrum governs how low the set point has to be to achieve a flat top and thereby how much light is discarded. From an efficiency standpoint it is more favorable to only have a small number of strong peaks dominating the dynamic range. In the setup itself, the component that would yield the highest efficiency improvement is the grating. The values in Table 1 are likely an overestimation since they are specified for the Littrow angle of $17.6 \mathrm{deg}$ and the light is actually incident on the grating at $24.5 \mathrm{deg}$ and about $5 \mathrm{deg}$. Therefore, an investigation into a more efficient grating choice could substantially improve efficiency.

\section{Summary}

Our LFC has a high dynamic range in its spectrum, which impedes calibration of an FTS. To alleviate this, we built a compact optical setup to control the spectral envelope of the LFC. With this setup, we obtain a spectrum that is flat over a wavelength range of over $200 \mathrm{~nm}$ in the nearinfrared. For $90 \%$ of this wavelength range, the dynamic range is below $3 \mathrm{~dB}$. The throughput of the setup with the activated control is about $2 \%$ to $3 \%$. The control loop remains stable over time scales much longer than a single FTS-measurement of up to $16 \mathrm{~min}$.

To our knowledge, this is the first demonstration of a spectrum control setup in the nearinfrared and the first measurement of a spectrally flattened LFC with an FTS. The manipulated LFC spectrum is sufficiently flat and has a high enough intensity to improve calibration of the FTS, with an increase of about $3 \%$ in the number of comb lines being found. However, the contamination and additional noise caused by the SLM modulation decrease the line fit quality. To deal with these issues, further investigation is necessary.

An astronomical echelle spectrograph would not suffer from these effects. This spectral flattening setup is therefore suited for calibration of a high-resolution grating spectrograph with an LFC and would improve calibration performance due to the reduced dynamic range and increased spectral stability.

\section{Acknowledgments}

P. Huke and M. Debus acknowledge funding from BMBF (projects No. 05A14MG3 and 05A17MG3). Parts of this manuscript were previously published in Ref. 16.

\section{References}

1. P. O. Schmidt, S. Kimeswenger, and H. U. Käufl, "A new generation of spectrometer calibration techniques based on optical frequency combs," in ESO Instrum. Calibration Workshop, pp. 409-412 (2007).

2. D. A. Braje et al., "Astronomical spectrograph calibration with broad-spectrum frequency combs," Eur. Phys. J. D 48(1), 57-66 (2008).

3. M. T. Murphy et al., "High-precision wavelength calibration of astronomical spectrographs with laser frequency combs," Mon. Not. R. Astron. Soc. 380(2), 839-847 (2007).

4. J. K. Ranka, R. S. Windeler, and A. J. Stentz, "Visible continuum generation in air-silica microstructure optical fibers with anomalous dispersion at $800 \mathrm{~nm}$," Opt. Lett. 25(1), 25-27 (2000).

5. T. A. Birks, W. J. Wadsworth, and P. S. J. Russell, "Supercontinuum generation in tapered fibers," Opt. Lett. 25(19), 1415-1417 (2000).

6. R. A. Probst et al., "Spectral flattening of supercontinua with a spatial light modulator," Proc. SPIE 8864, 88641Z (2013).

7. R. A. Probst et al., "Spectrally flattened, broadband astronomical frequency combs," in Conf. Lasers and Electro-Opt., IEEE, pp. 1-2 (2015).

8. A. Monmayrant, S. Weber, and B. Chatel, "A newcomer's guide to ultrashort pulse shaping and characterization," J. Phys. B: At. Mol. Opt. Phys. 43(10), 103001 (2010). 
9. A. M. Weiner, "Ultrafast optical pulse shaping: A tutorial review," Opt. Commun. 284(15), 3669-3692 (2011).

10. G. Lazarev et al., "LCOS spatial light modulators: trends and applications," in Optical Imaging and Metrology: Advanced Technologies, W. Osten and N. Reingand, Eds., pp. 129, John Wiley \& Sons, Ltd, Hoboken, New Jersey (2012).

11. P. Huke et al., "Characterization and calibration of a Fourier-transform spectrometer using a laser frequency comb," J. Opt. Soc. Am. B 36(7), 1899-1906 (2019).

12. C. A. Palmer and E. G. Loewen, Diffraction Grating Handbook, Vol. 5, Thermo RGL, New York (2002).

13. S. Schäfer et al., "Fiber-coupling of Fourier transform spectrographs," Proc. SPIE 11447, 114473Q (2020).

14. D. Meyer, "Gamma curve calibration of a spatial light modulator," Bachelor's thesis, Göttingen University (2019).

15. S. K. Kalyoncu et al., "Analytical study on arbitrary waveform generation by mems micro mirror arrays," Opt. Express 20(25), 27542-27553 (2012).

16. M. Debus et al., "Spectral envelope control for a flat frequency comb spectrum," Proc. SPIE 11451, 1144-1152 (2020).

Biographies of the authors are not available. 